at about $38 \times 10^{-5} \mathrm{~cm} \cdot 3 / \mathrm{sec} . / \mathrm{cm}$. and then began to decrease slowly, presumably because evaporation of the liquid inside the Dewar flask was not entirely prevented by the ground joint. Preliminary measurements have shown that the maximum rate of flow is a function of the height of the film, being only about $26 \times 10^{-5} \mathrm{~cm} \cdot{ }^{3} / \mathrm{sec} . / \mathrm{cm}$. for a film of height $4 \mathrm{~cm}$. In order of magnitude these rates of flow agree satisfactorily with the data of Fig. 1 .

It is worth noticing that saturation occurs at a temperature difference of approximately $10^{-3} \circ \mathrm{K}$. which, according to H. London' ${ }^{5}$ thermodynamic formula, corresponds to a thermo-mechanical pressure head of only $3 \mathrm{~cm}$. of helium at this temperature. When no electrical power was supplied, a transfer of $12 \times 10^{-5} \mathrm{~cm} .3 / \mathrm{sec}$. $/ \mathrm{cm}$. was observed and was probably caused by unwanted heat inflow to the system. It was not possible, however, to decide whether this transfer took place under zero temperature difference or whether it required a temperature difference of the order of $10^{-4} \circ \mathrm{K}$.

I should like to thank Prof. J. F. Allen for valuable help and discussion.

Royal Society Mond Laboratory,
Cambridge.

K. R. ATKINS

April 28.

1 Rollin, B. V., Actes du $77^{\circ}$ Cong., intern. du Froid. La HayeAmsterdam, 1, 187 (1939).

'Daunt, J. G., and Mendelssohn, K., Proc. Roy. Soc., A, 170, 423, 439 (1939).

${ }^{3}$ Bijl, A., de Boer, J., and Michels, A., Physica, 8, 655 (1941).

- Daunt, J. G., and Mendelssohn, K., Nature, 148, 719 (1939).

London, H., Proc. Roy. Soc., A, 171, 484 (1939).

\section{Diffraction of Light by Very High Frequency Ultrasonic Waves}

IN two recent notes ${ }^{1}$, we reported that ultrasonic waves of frequencies as high as $100 \mathrm{Mc}$./sec. were generated. The diffraction patterns produced by such waves were found to exhibit interesting features. Since then, by using a tourmaline plate and specially designed circuits, we have been able to push up this frequency to nearly $180 \mathrm{Mc}$. $/$ sec., and to study the diffraction effects produced when progressive waves of such high frequencies are maintained in a column of water. Results for the velocity of sound, measured at two different frequencies, are given below. In obtaining these, it has been assumed that the velocity in water is 1,504 metres per second at $15 \mathrm{Mc} / \mathrm{sec}$. and $26.5^{\circ} \mathrm{C}$. If the slightly higher temperature of the medium when the highest frequency is used is taken into account, it will be noticed that there is no dispersion of ultrasonic velocity.

It is well known that the first-order lines on both sides are easily obtained at normal incidence when low frequencies are used, and we have found in our earlier work that this feature persists even at $50 \mathrm{Mc}$./sec. Moreover, when the crystal is tilted, the line on the appropriate side increases in intensity

\begin{tabular}{|c|c|c|c|c|c|}
\hline $\begin{array}{l}\text { Order of } \\
\text { harmonic }\end{array}$ & $\begin{array}{c}\text { Frequency, } \\
\text { (megacycles } \\
\text { per sec.) }\end{array}$ & $\begin{array}{c}\text { Fringe } \\
\text { width, } d \\
\text { (cm.) }\end{array}$ & $v / d$ & $\begin{array}{l}\text { Velocity } \\
\text { (metres } \\
\text { per sec.) }\end{array}$ & $\begin{array}{l}\text { Temp. } \\
\left({ }^{\circ} \mathrm{C} .\right)\end{array}$ \\
\hline $\begin{array}{c}9 \\
83 \\
107^{*}\end{array}$ & $\begin{array}{c}15 \cdot 29 \\
140 \cdot 9 \\
177 \cdot 2\end{array}$ & $\begin{array}{l}0 \cdot 1237 \\
1 \cdot 140 \\
1 \cdot 441\end{array}$ & $\begin{array}{l}123 \cdot 6 \\
123 \cdot 6 \\
122 \cdot 9\end{array}$ & $\begin{array}{l}1504 \\
1504 \\
1511\end{array}$ & $\begin{array}{l}26 \cdot 5 \\
26 \cdot 5 \\
27 \cdot 9\end{array}$ \\
\hline
\end{tabular}

* The plate distance in this case is slightly different from that adopted in the other two. and attains a maximum at the corresponding reflexion angle. The line on the opposite side decreases in intensity but generally does not vanish altogether, showing that if we regard the phenomenon as a reflexion, the angular region in which it occurs when moderate frequencies are used is not at all sharp. In the present experiments, we have made special arrangements to reduce the power supplied to the crystal at moderate frequencies to such an extent that the first-order diffraction lines obtained are as weak as those obtained at $177 \cdot 2 \mathrm{Mc}$./sec. Even under such conditions, both lines appear at normal incidence and they exist simultaneously over practically the entire range as the tilt of the sound wavefront is varied from the reflexion position on one side to the reflexion position on the other.

On the other hand, the diffraction patterns at the very high frequencies employed disappear altogether when the sound wave is exactly normal to the incident light. The crystal holder has to be tilted to the correct angle on one side or the other so that the first-order diffraction line on that side may appear. At these high frequencies, the first-order lines on both sides are never present simultaneously. It may even be said that the appearance of the first-order line is quite critical. The tilt angle measured in our experiments with different frequencies has been found to be in agreement with the value derived from the equation $\lambda / \lambda^{*}=2 \mu \sin 0$. In accordance with this formula, we find that the sound wave-front has to be tilted from the normal position by $1^{\circ} 29^{\prime}$ in the case of $177.2 \mathrm{Mc} / \mathrm{sec}$. in order that the first-order line on one side may appear. So small a deviation as about $8^{\prime}$ from this position results in complete disappearance of the line. The wave-length of sound in water corresponding to $177.2 \mathrm{Mc} / \mathrm{sec}$. employed in the present investigation is $0.000849 \mathrm{~cm}$., which is only about fourteen times as large as the wave-length of sodium light.

\section{S. Bhagavantam}

B. RaMaChaNdRa RAO

\section{Andhra University,}

WaItair, India.

Feb. 15.

${ }^{1}$ Nature, 158, 484 (1946); 159, 266 (1947).

\section{A New Hot-Wire Vacuum Gauge}

IT is known that the range of a hot-wire vacuum gauge may be extended to $10 \mathrm{~mm}$. mercury pressure or above, if by some means the current supplied to

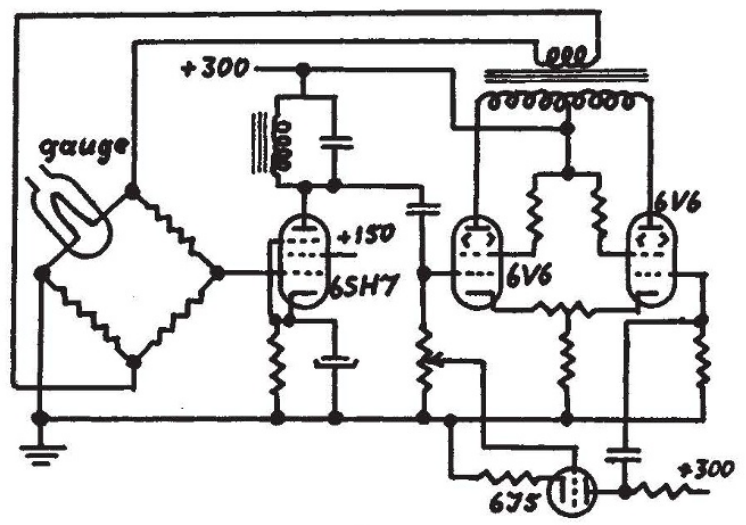

Fig. 1 